\title{
Triple Play Service under the Impact of Non Stationary Noise in a DSL System: an Amazon Approach
}

\author{
Lamartine V. de Souza ${ }^{a}$, Diego Cardoso ${ }^{a}$, Marcelino Silva ${ }^{a}$, Marcos Seruffo $^{a}$, Carlos R. L. \\ Francês $^{a}$, João C. W. A. Costa ${ }^{a}$, Agostinho L. S. Castro ${ }^{a}$, Gervásio Cavalcante ${ }^{a}$, and Jaume \\ Rius i Riu ${ }^{b}$ \\ ${ }^{a}$ Federal University of Pará (UFPA), Rua Augusto Corrêa no 01, Guamá, Belém/PA, Brazil. \\ ${ }^{b}$ Ericsson AB, Stockholm, Sweden.
}

\begin{abstract}
The Brazilian Amazon has sui generis characteristics that affect strongly the communication technologies, such as high humidity and temperature. These characteristics cause impact in the existent infrastructure, especially in twisted-pair copper lines. At the moment, new services are based on multimedia applications, as voice over internet protocol (VoIP), video on demand (VoD), and internet protocol television (IPTV). Such services use digital broadband networks such as ADSL2+ (Asymmetric Digital Subscriber Line) to transmit the data. Broadband services require data rates which can only be achieved by using relatively high spectrum frequencies. At high frequencies the DSL signal is more susceptible to external noise sources, such as radio frequency interference and impulsive noise. For this purpose, an experimental setup has been built at UFPA that consists of noise generator, traffic generator, real cables, modems and DSLAM (digital subscriber line access multiplexer). This paper aims at characterizing how the noise impacts on triple play services for a broadband system using a DSL loop on Amazon environment. The objective of the noise impact experimentation is to observe the behavior of a DSL system under more realistic but controlled line conditions. Metrics as lost packet, jitter, latency, and throughput are used to characterize the triple play service in a DSL loop under the noise impact. Through the real experiments and controlled loop conditions, this paper allow identify, from application level point of view, how robust DSL system is in respect to noise occurrence. Additionally, it is described a methodology for noise impact measurements using a DSL system.
\end{abstract}

Keywords: broadband networks, IPTV, DSL systems, noise measurements, test procedures

\section{INTRODUCTION}

The growing dissemination of information via digital mediums throughout the world brings new realities to the surface such as new technologies, strengthening the feeling of dynamism in the information diffusion process and also improving the quality of life of its population. In order to supply a true increase in services to the citizen and/or to provide its social/digital inclusion in regions of typical, end-user low availability infrastructure, including geographical specificities, it is vital to take note of prior studies that represent the cost/benefit relation that is favorable to specified telecommunications solutions.

It seems appropriate therefore that applications such as teleducation, considered to be strategically important to the region, are considered impractical in many situations because of the difficulties in providing access to the end-user, usually beneficial as an effective educational resource. This process makes these hidden concerns more evident in unsupported areas which are in the most need of access technologies (in this case, those areas synonymous with "last-mile technology").

Any digital/social inclusion action must have previously gone through a process in which it is necessary to previously establish the access infrastructure. However, this application feasibility process will be compromised if this process is overlooked or omitted. In this context, there must be alternative proposals in order to include those regions that are deprived of basic infrastructure. The Amazon region is a specific example of this lack

Further author information: (Send correspondence to Lamartine V. de Souza) E-mail: lvsouza@ufpa.br, www.lea.ufpa.br/lamartine, Telephone: +55 9132017740

Broadband Access Communication Technologies II, edited by Raj Jain, Benjamin B. Dingel, Shozo Komaki, Shlomo Ovadia Proc. of SPIE Vol. 6776, 67760K, (2007) · 0277-786X/07/\$18 - doi: 10.1117/12.734756

Proc. of SPIE Vol. 6776 67760K-1 
of infrastructure, this being as much in relation to the backbone as it is in respect to the last-mile technology. However, as a rule, defined solutions that have already been concluded from other parts of the country do not perfectly adhere to that of the Amazon in relation to specificities presented in this region.

Currently, IPTV technology (Internet Protocol Television) has been the focus of high levels of research, this being related to the development of computer networks, evolved with the intention of increasing the breadth of communication as well as to establish transmission guarantees, therefore, further establishing the concept of QoS (Quality of Service). This scenario encapsulates a large rivalry between: telecommunications companies (telcos) and Cable TV companies (CATV), where the telcos see IPTV as an opportunity to maintain their current market position ${ }^{1} 2$. Another line of research is via frameworks, developed in order to optimize the quality of transmission and the end-user experience ${ }^{3}{ }^{4} 5$. In El-Sayed et al. ${ }^{6}$ an IPTV case study was carried out over technologies such as fiber optic, SONET and xDSL, utilizing simulation tools only and therefore not establishing specific xDSL parameters. Seong et al. ${ }^{7}$ has presented some appropriate measures for the evaluation of IPTV technologies.

This work touched on some of the access technologies for digital inclusion, retouching on typical Amazon scenarios and how to apply them to IPTV, that is, television connected to the Internet. The second topic touched on the subject of IPTV and the services it offers. The third topic showed state-of-the-art current access technologies, used for the provisioning of services, with emphasis on xDSL and its advantages. In the fourth topic we have a description of the scenario and the tools used for this testing, followed by the fifth topic that shows the results obtained from these experiments, conclusions shown in the topic that follows it.

\section{IPTV}

IPTV is a high velocity bilateral internet service that uses a connection via IP packets (Internet Protocol), sending images at a defined standard level and providing a triple play service (voice, video and audio), including data streaming 783 .

The IPTV service allows the use of a broadband network connection in order to deliver TV content that potentially has the addition of interactive services. Current IP networks offer a packet delivery service that is called "best effort" that does not guarantee performance to its end-users, signifying therefore the potential loss of data during transmission. Traditional applications such as (WWW, e-mail, file transfer etc) require delivery reliability and guarantees via the use of the Transmission Control Protocol (TCP). These are called "elastic" applications, since they do not require a minimum transmission capacity or a maximum lag in order to function correctly.

Delay, lost packets and the network transmission capacity are very important considerations for IPTV applications due to the fact that these networks can only tolerate small packet losses, this resulting in severe temporization or minimum transmission capacity restrictions in order to guarantee its own viability.

\section{ACCESS TECHNOLOGIES}

Access to communications services in the Amazon region are not provided by a single technology, but by a combination of technologies, these generating an innumerable amount of problems such as: differences between bandwidths, service qualities, reliability and scalability. Because of this it is fundamental that a group of support tools are readily available in order to make planning and deployment of existing technologies possible.

Currently there exists many forms of last-mile technologies, these being divisible into two categories: (a) cable technologies: fiber optics (for access but not for backbone); PLC (Powerline Communication); CATV; xDSL and dialed line; (b) wireless access technologies: satellites, cellphone systems and local wireless networks (WLANs).

It is therefore necessary to investigate the access technology utilization viability, taking note of imposed restrictions in typical Amazon scenarios, focusing on capacity planning, optimization and performance evaluation issues. The intention is to contribute to this with a series of digital inclusion actions that require the minimum access infrastructure possible.

DSL has a high level of world penetration, these being considered the most dominant broadband technologies, not only in Europe, but also in Latin America and in developing countries such as India ${ }^{9} 10^{11}$. DSL technology 
is responsible in Latin America for around $77 \%$ of all current broadband access, whereas in Brazil, this value is as high as $85 \%$. In quantitative terms, at the end of 2005 there were almost 5,300,000 Latin American ADSL technology sign-ups ${ }^{10}$.

In spite of such high numbers, the penetration of broadband services in Latin American residencies is estimated to be at only $9 \%$ for the 2006 year end, being projected at $17 \%$ by the end of $2015^{10}$. It is comprehendible then that there is a long road to traverse before broadband services are widely used in Latin America.

Considering that DSL systems have been affirmed as a technology base for broadband systems, an adequate study into harmful effects on system performance (stationary noise and non-stationary noise, electromagnetic interference, etc.) has become necessary.

\section{SCENARIO}

So that this last-mile technology analysis is able to be carried out, a typical IPTV transmission scenario is utilized. In this system there will be machines communicating with triple play traffic - a service made available via IPTV - a convergence between voice, video and data ${ }^{8}$. The utilized test scenario is composed of modems, DSLAM, telecommunications cables, noise generator and computers - the experiment being assembled at the LabIT (Telecommunications Technology Innovation Lab) at the UFPA. Figure 1 shows the test scenario.

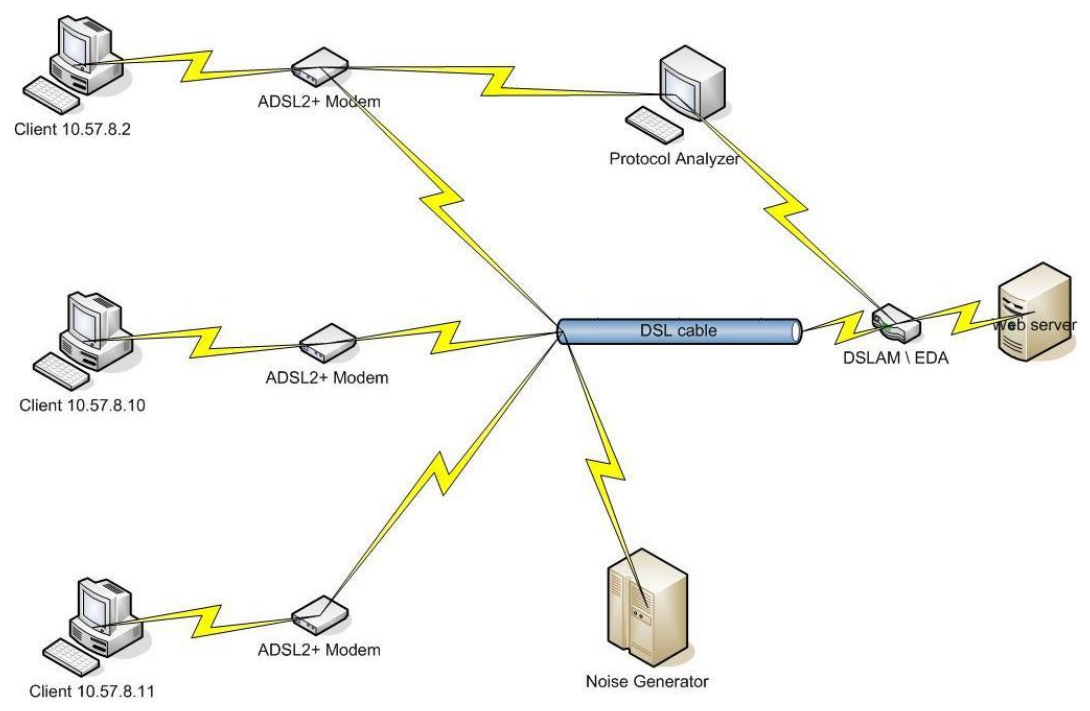

Figure 1. Utilized test scenario.

The generation of impulsive noise is made by a DSL 5500 noise generator from Spirent Communications, the noise being generated in the ADSL operating band from between $4.3125 \mathrm{kHz}$ to $1.104 \mathrm{MHz}$. A RADCOM ${ }^{12}$ protocol analyzer was also used, its function to filter the packets that would be transmitted around the network, isolating specific flux data sets in order to measure performance.

The combination of DSLAM / EDA (Ethernet DSL Access) comprises of existing central telephonic equipment, thus allowing data to be communicated via a marriage of DSL equipment. The computer connected to the DSLAM network is responsible for the creation of video flux data that will be distributed via multicast between the clients.

Data access was provided by utilizing physical metallic cables which are readily available around the Computing and Electrical Engineering Laboratory building at the UFPA. Utilization of these cables allowed for higher reliability in gathered results since they are subject to the same specific external influences from the Amazon region such as heat, high humidity and rain, as well as the normal working conditions that they are submitted to on a daily basis such as: connectors along the cables, bends, external noise influences, etc. 
In order to pre-determine the length of cables to be used, the Telebrás standard Brazilian law - 225-540-788 (April 1997), proposing a distance of 2,800 meters for link testing was utilized. However, in this experiment 2,750 meters were used because of the fact that only 250 and 500 meter cable length sizes were available.

The noise used in the tests is denominated as "impulsive noise". Such types of noise contain non-stationary characteristics and are derived from spurious emissions that come from radio stations, car combustion and atmospheric discharges. The noise that was utilized in the tests originates from experiments carried out by BT/DT (British Telecom / Deutsch Telekom) studies ${ }^{13}$. In these studies, a noise model was developed by using the measures that were taken from telephone installations in England and Germany. Even though this noise model was carried out using measures from other countries, the non-stationary aspect characteristics in terms of amplitude, duration and time intervals have become universally utilized because of their general character.

For the creation of data traffic, a benchmark called SIEGE ${ }^{14}$ was used. It utilizes free software (versions for Linux and UNIX) in order to carry out access tests, establishing a benchmark for the HTTP (Hypertext Transfer Protocol). A simulation is conducted on a server that determines the number of connections per second during a pre-determined time period.

For the generation of video traffic, the VLC ${ }^{15}$ (VideoLAN Client) was used, this being a multimedia reproducer that supports various video as well as various streaming protocols. For the video test, a VLMS (VideoLAN MiniServer) ${ }^{15}$ was configured, as well as a VLC streaming server that generates multicast traffic, transmitting at a rate of $1 \mathrm{Mbps}$ over the three machines (video with MPEG2 codec). Callgen was used in order to transmit voice. This is a VoIP tool, developed by the OpenH232 project ${ }^{16}$, that is widely used for conducting tests ${ }^{17}$.

\section{RESULTS}

By using the tools described above, tests were carried out in which a video file (using the MPEG2 codec) of approximately 15 minutes was transmitted 2 times in order to complete 30 minutes of testing. Besides this, Callgen was also used in order to create VoIP calls between 2 client machines, as well as another VoIP call between a client machine and a video/HTTP server - these calls being repeated a total of 10 times. Each call contained an $81 \%$ characteristic of audio data and a $20.38 \%$ characteristic of silent data. The voice file was obtained in Podopera ${ }^{18}$. The HTTP requests were generated via the SIEGE benchmark utility, in which 5 HTTP requests were carried out simultaneously in simplified form. This is equivalent to each user machine opening 5 browser sessions.

VoIP communication can be analyzed over 2 principal parameters: jitter (network characteristics) and MOS - a benchmark that is utilized in order to define communication quality ${ }^{17}$. Call classification is found in Table 1 together with the points attributed to each classification.

Table 1. MOS (Mean Opinion Score) Classification.

\begin{tabular}{c|c|c}
\hline MOS & Quality & Degree of interference \\
\hline 5 & Excellent & Imperceptible \\
4 & Good & Perceptible but not incommodious \\
3 & Fair & Acceptable \\
2 & Poor & Incommodious \\
1 & Bad & Very incommodious \\
\hline
\end{tabular}

Various tests were carried out in which the BT/DT noise was introduced and gradually increased from its initial power of $-24.2 \mathrm{dBm}$ (in steps of $2 \mathrm{~dB}, 4 \mathrm{~dB}, 6 \mathrm{~dB}$ and $8 \mathrm{~dB}$ ). This method allows for the study of application behavior by introducing a specific noise level. Tables 2, 3 and 4 show the obtained results for VoIP, HTTP and Video traffic, respectively.

\begin{tabular}{c|c|c|c|c|c}
\multicolumn{6}{c}{ Table 2. Values obtained from VoIP. } \\
\hline & No noise & $2 \mathrm{~dB}$ & $4 \mathrm{~dB}$ & $6 \mathrm{~dB}$ & $8 \mathrm{~dB}$ \\
MOS & 3.74 & 3.72 & 3.69 & 3.73 & 3.76 \\
Jitter Average (s) & 9.75 & 9.75 & 9.57 & 9.85 & 9.42 \\
\hline
\end{tabular}


Table 3. HTTP - Values obtained by SIEGE.

\begin{tabular}{c|c|c|c|c|c}
\hline & No noise & $2 \mathrm{~dB}$ & $4 \mathrm{~dB}$ & $6 \mathrm{~dB}$ & $8 \mathrm{~dB}$ \\
Delay(s) & 0.121 & 0.1194 & 0.1192 & 0.1203 & 0.1202 \\
Jitter(s) & 0.001 & 0.001 & 0.001 & 0.001 & 0.001 \\
Lost Packets(\%) & 0.34454 & 0.341272 & 0.337632 & 0.339985 & 0.343947 \\
\hline \multicolumn{7}{c}{ Table 4. Values obtained by video. } \\
\hline Delay(s) & No noise & $2 \mathrm{~dB}$ & $4 \mathrm{~dB}$ & $6 \mathrm{~dB}$ & $8 \mathrm{~dB}$ \\
Lost Packets(\%) & 0.01624 & 0.016356 & 0.016385 & 0.017346 & 0.019433 \\
\hline
\end{tabular}

All of the above measurements were obtained by using the RADCOM protocol analyzer, this tool already having supplied VoIP measurements via a specific program called MediaPro. Via a trace archive created by the RADCOM $\AA$ analyzer, programs were implemented that generated the other presented metrics. Further information about data analyzed by RADCOM® and MediaPro can be obtained in Radcom ${ }^{12}$.

From Tables 2, 3 and 4, it is perceivable that the MOS and jitter values suffer significant alterations by the gradual increase in noise intensity, the above values remaining at acceptable values for adequate communication.

Video suffers a certain amount of degradation and an increase in delay, together with an increase in noise intensity. This fact can also be observed in HTTP behavior which does not have large alterations in delay either, but which does have more significant lost packet than that of video. The variation in jitter is very low. This can be observed in Fig. 2 to Fig. 6. In Fig. 2 and 3, the scale vary between 8 and -8 seconds, whereas in Figures 4 , 5 and 6 the variations are larger and more intense.

\section{CONCLUSION}

IPTV is a promising technology for the utilization of broadband services in television and other media services over a secure link. This work was directed at carrying out an analysis of one of the possible available access technologies for IPTV applications in regions that do not possess a privileged capacity infrastructure. It is possible to start an operation in order to implement extrapolations through the most appropriate real or simulated situations by utilizing new DSL parameters that are specific to the Amazon region.

It is further intended that this operation will establish methods that can promote digital inclusion into the percentage of the population that is still lacking in even primitive resources. The utilization of IPTV will bring benefits, such as: the right to vote, easy issue of documents (Tax documents, Ballot cards, etc), higher speed and accuracy in the taking of censuses, distance learning (DL), disease prevention campaigns, as well as other advantages that have emerged with the deployment of this service.

\section{ACKNOWLEDGMENTS}

Authors would like to thank the Research and Development Centre, Ericsson Telecomunicacões S.A., Brazil, Federal University of Pará, Department of Electrical and Computing Engineering, Belém, Pará, Brazil. And Ericsson AB, Access Signal Processing Laboratory, Älvsjö, Sweden. Some of the authors want to acknowledge the financial support received from the European Commission IST 6th Framework and from the Swedish Agency for Innovation Systems, VINNOVA, through the IST - MUSE and the Eureka - Celtic BANITS projects respectively, which partially enabled this work.

\section{REFERENCES}

1. Intel, "H.264 \& IPTV over DSL, Enabling new Telco Revenue Opportunities," 2006.

2. Cisco, "Telco Deploys Enhanced IPTV and Ethernet Service - White Paper," 2006.

3. J. Wu, K. Peng, M. Lu, C. Lin, Y. Cheng, P. Huang, J. Yao, and H. Cheng, "HotStreaming: Enabling Scalable and Quality IPTV Services," in IPTV Workshop, International World Wide Web Conference, May 2006. 
4. H. X. Zhang, J. Liu, B. Li, and T. S. P. Yum, "DONet/CoolStreaming: A Data-driven Overlay Network for Live Media Streaming," June 2004.

5. C. H. Muntean and G. Muntean, "Framework for Interactive Personalised IPTV for Entertainment," in W3C WWW Conference, May 2006.

6. M. El-Sayed, H. Ying, S. Kulkarni, and N. Wilson, "Access transport network for IPTV video distribution," in Optical Fiber Communication Conference and the 2006 National Fiber Optic Engineers Conference, 2006.

7. H. K. Seong and H. L. Gil, "The Study on SLA Metrics and System Architecture for IP TV," in 8th International Conference on Advanced Communication Technology (ICACT2006), February 2006.

8. A. Al-Hezmi, Y. Rebahi, T. Magedanz, and S. Arbanowski, "Towards an Interactive IPTV for Mobile Subscribers," in ICDT/IEEE International Conference on Digital Telecommunications, 2006.

9. B. Olsen, D. Katsianis, D. Varoutas, K. Stordahl, J. Harno, N. Elnegaard, I. Welling, F. Loizillon, T. Monath, and P. Cadro, "Technoeconomic Evaluation of the Major Telecommunication Investment Options for European Players," in IEEE Network, pp. 6-15, July/August 2006.

10. D. Arenas, C. Caldas, C. Ramundo, S. Vargas, and L. Hostos, "Challenges to expanding Fixed Broadband Services in Latin America - Alcatel White Paper," September 2006.

11. V. Faudon, D. Vleeschauwer, E. Festraets, and P. Ross, "End-User Services for Broadband uptake in HighGrowth Economies - Alcatel White Paper," September 2006.

12. Radcom, "The state of art," 2007.

13. I. Mann, S. McLaughlin, W. Henkel, R. Kirkby, and T. Kessler, "Impulse Generation with Appropriate Amplitude, Length, Inter-Arrival, and Spectral Characteristics," in IEEE Journal on Selected Areas in Communications, pp. 901-912, June 2002.

14. Siege, "www.joedog.org/joedog/siege," January 2007.

15. VLC, "VLC Media PLayer," January 2007.

16. OpenH323, "http://www.openh323.org," January 2007.

17. J. A. M. de Souza, M. S. da Silva, C. R. L. Francês, J. C. W. A. da Costa, M. E. V. Segatto, F. R. Antônio, and G. Rodrigues, "A feasibility study of powerline communication technology for digital inclusion in Brazilian Amazon," in SPIE OPTICS EAST 2006, September 2006.

18. Podopera, "http://podopera.co.uk," 2007.

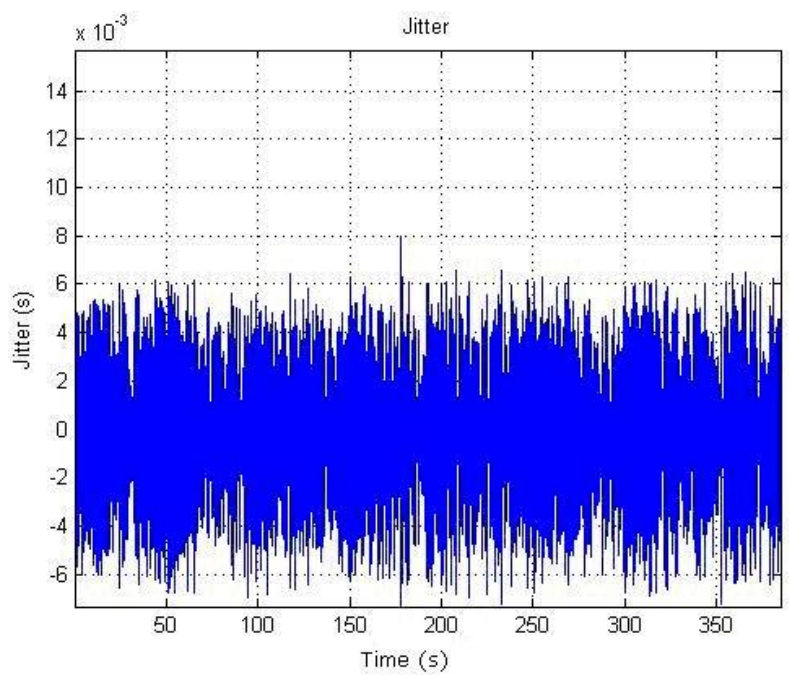

Figure 2. Jitter behavior without noise. 


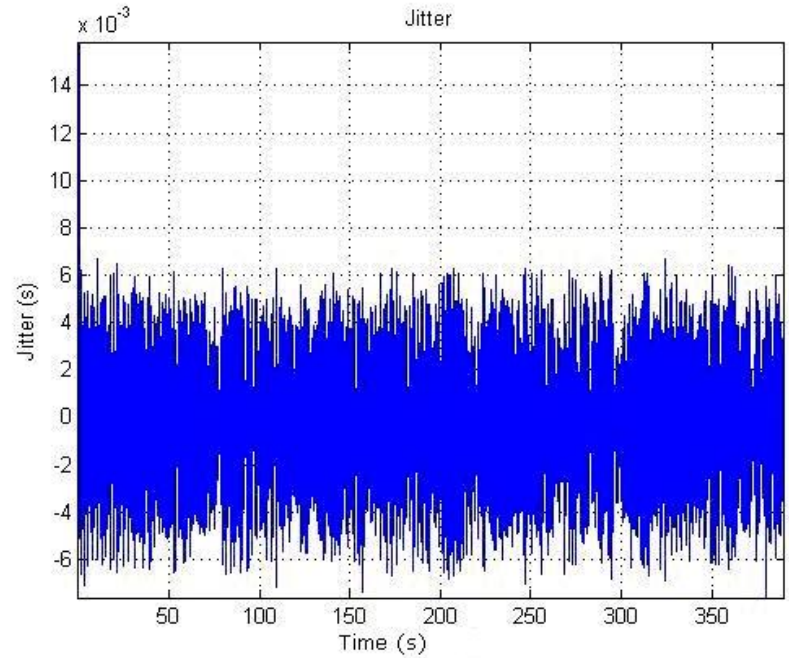

Figure 3. Jitter behavior with 2 dB.

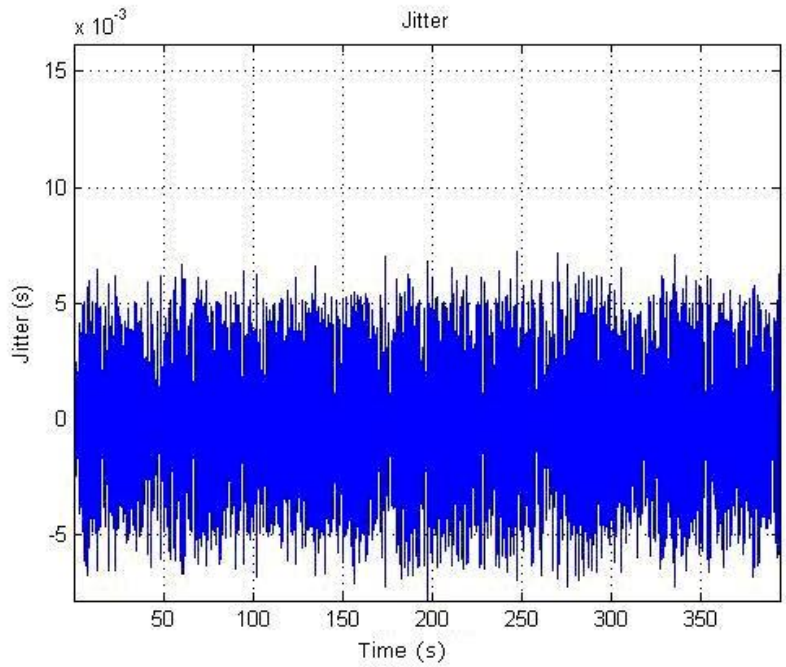

Figure 4. Jitter behavior with $4 \mathrm{~dB}$. 


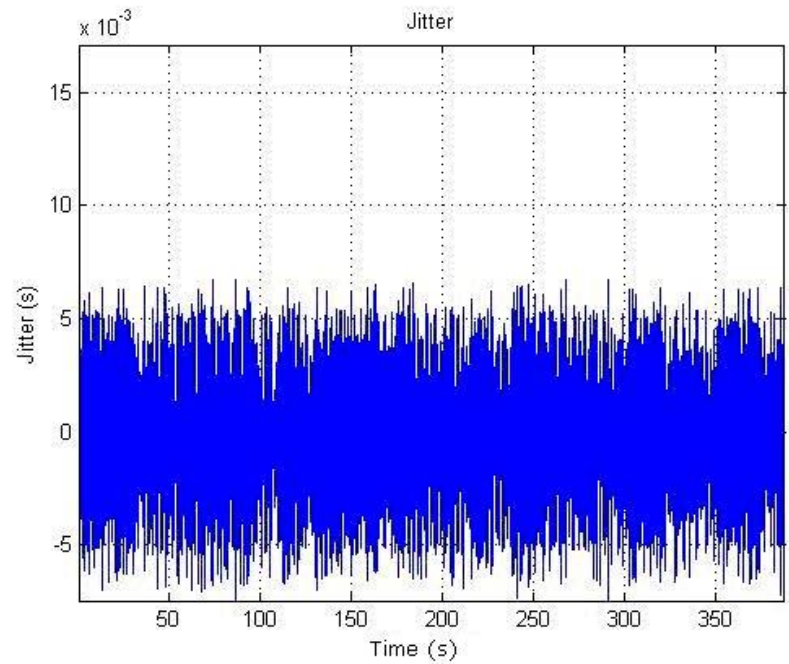

Figure 5. Jitter behavior with 6 dB.

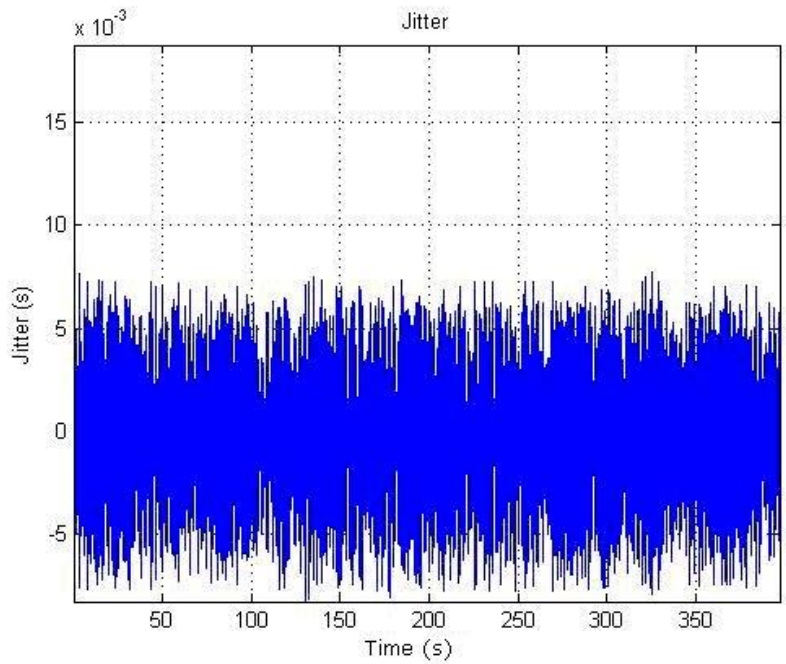

Figure 6. Jitter behavior with $8 \mathrm{~dB}$. 